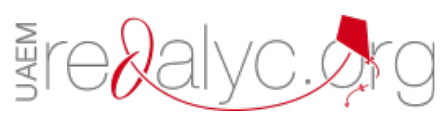

Centro Sur

ISSN: $2600-5743$

compasacademico@icloud.com

Grupo Compás

Ecuador

\title{
Hacia la integración de los sistemas de gestión: calidad y seguridad y salud en el trabajo
}

Sotolongo Sánchez, Maria

Hacia la integración de los sistemas de gestión: calidad y seguridad y salud en el trabajo

Centro Sur, vol. 2, núm. 1, 2018

Grupo Compás, Ecuador

Disponible en: http://www.redalyc.org/articulo.oa?id=588861692004 


\title{
Hacia la integración de los sistemas de gestión: calidad y seguridad y salud en el trabajo
}

Towards the integration of management systems: quality and safety and health at work

\author{
Maria Sotolongo Sánchez marisotolongo@gmail.com \\ Universidad Auntónoma de Barcelona, España \\ http://orcid.org/0000-0002-8341-7455
}

Centro Sur, vol. 2, núm. 1, 2018

Grupo Compás, Ecuador

Recepción: 16 Agosto 2017 Aprobación: 11 Abril 2018

Redalyc: http://www.redalyc.org/ articulo.oa?id $=588861692004$
Resumen: En el contexto actual se necesita de sistemas de gestión que permitan controlar de forma sistemática las actividades y procesos de la empresa, con la participación e implicación de todos sus trabajadores, con el objetivo de lograr los resultados previstos. Sin embargo, las actuales exigencias en materia de calidad y seguridad y salud en el trabajo, relativas a la satisfacción de los trabajadores, de los clientes y de las demás partes interesadas, han demostrado que los sistemas de gestión son grandes aliados de las organizaciones que quieren mejorar su desempeño en estas áreas. En este sentido, muchas organizaciones para optimizar su gestión optan por integrarlos. Como una contribución a esta problemática, el objetivo de esta disertación es reflexionar sobre la necesidad de sistemas integrados de gestión de calidad y seguridad y salud en el trabajo ante las exigencias del marco legal de la ISO 9001:2015 y la ISO 45001:2018.

Palabras clave: sistemas integrados de gestión, calidad, seguridad y salud en el trabajo.

Abstract: In the current context, management systems are needed to systematically control the activities and processes of the company, with the participation and involvement of all its workers, in order to achieve the expected results. However, the current demands in terms of quality and safety and health at work, concerning the satisfaction of workers, customers and other interested parties, have shown that management systems are great allies of organizations that They want to improve their performance in these areas. In this sense, many organizations to optimize their management choose to integrate them. As a contribution to this problem, the objective of this dissertation is to reflect on the need for integrated systems of quality management and safety and health at work in view of the requirements of the legal framework of ISO 9001: 2015 and ISO 45001: 2018.

Keywords: integrated management, quality, safety and health at work systems.

\section{INTRODUCCIÓN}

El nuevo paradigma de exigencia respecto a la competitividad unido a los cambios en el marco legislativo y normativo a escala internacional en materia de gestión de la calidad y seguridad y salud en el trabajo, obliga a las empresas a adaptarse a las anteriores exigencias e implantar sistemas de gestión que permitan que sus productos o servicios tengan un valor agregado que sean bien vistos, den confianza y favorezcan la decisión de compra por los clientes. A ello contribuyen los Sistemas de Integrados de Gestión (SIG), enfocados al logro de unos determinados resultados, en relación con los objetivos de la Calidad y la Seguridad y Salud en el 
Trabajo, para satisfacer las necesidades, expectativas y requisitos de las partes interesadas, según corresponda.

Un Sistema de Gestión es un conjunto de elementos mutuamente relacionados o que interactúan, para establecer la política y los objetivos, y para lograr dichos objetivos. Luego entonces, un Sistema Integrado de Gestión de Calidad y Seguridad y Salud en el Trabajo posibilita y simplifica la implantación de un único sistema de gestión eficaz, adecuado para la empresa.

El origen y necesidad de estos sistemas ha sido diferente: la calidad se ha desarrollado impulsada fuertemente por la competencia y por la necesidad de mejorar la competitividad empresarial y la seguridad por el establecimiento de regulaciones gubernamentales y por la presión de las organizaciones sindicales para garantizar condiciones de trabajo seguras. A pesar de esto se asume desde un mismo enfoque de gestión basado en el ciclo Deming (Planear

- Hacer - Verificar - Actuar) y la mejora continua que implica:

(1) Planificar el desempeño y programar las actividades a realizar mediante el establecimiento de políticas, objetivos, indicadores, metas y programas a lograr, así como la definición de responsabilidades asociadas al desarrollo de los procesos y al cumplimiento de las tareas.

(2) Ejecutar las actividades según lo programado mediante la aplicación de métodos, técnicas y procedimientos establecidos.

(3) Controlar el cumplimiento de la ejecución tanto a nivel operativo (control y seguimiento en los procesos) como estratégico mediante las revisiones del sistema fundamentalmente aunque para algunos de los sistemas se utilizan otras formas de control.

(4) Mejora continua del desempeño tomando acciones correctivas y preventivas para solucionar y evitar no conformidades.

En este sentido, cualquiera que sea el Sistema Integrado de Gestión que se adopte, debe contemplar teniendo en cuenta la estructura de alto nivel de las normas los requisitos siguientes: Contexto de la organización, Liderazgo, Planificación, Soporte, Operación, Evaluación del desempeño y Mejora.

Sin dudas, la reciente publicación de la ISO 45001:2018 va a facilitar aún más la integración de estos sistemas. Como una contribución en este sentido, el objetivo de esta disertación es reflexionar sobre la necesidad de Sistemas Integrados de Gestión de Calidad y Seguridad y Salud en el Trabajo ante las exigencias del marco legal de la ISO 9001:2015 y la ISO 45001:2018, sus ventajas y desventajas y recomendaciones para su integración.

El concepto de sistema de gestión integrado surge como una forma en que una organización puede asegurarse de que se cumpla de manera eficaz y eficiente con requisitos de varios sistemas de gestión. El sistema integrado de gestión puede cubrir los aspectos de la gestión institucional, la calidad del producto, el servicio al cliente, el desempeño en SST; entre otros aspectos. 


\section{MATERIALES Y MÉTODOS}

Los elementos comunes de los diferentes aspectos de la organización brinda una oportunidad obvia para aumentar la eficacia y eficiencia integrándolos. Las organizaciones tienen un equipo de dirección y parece lógico adoptar un Sistema de Gestión único que abarque varias metodologías necesarias para cumplir las responsabilidades de la Dirección y los objetivos de la institución. Muchas organizaciones que ya tienen implementado algún Sistema de Gestión ven las similitudes existentes con otros y reconocen que mantener la multiplicidad no resulta práctico ni económico. Así la integración de los sistemas puede tener ventajas como:

- Se satisfacen las necesidades y expectativas de las partes interesadas al establecer la obligatoriedad de cumplir al mismo tiempo con los requisitos legales y regulatorios de calidad y de seguridad y salud en el trabajo especificado y otros.

- Mejora la eficacia y la eficiencia de los procesos, aumentando la consistencia, la trazabilidad, evitando las redundancias y las incoherencias.

- Se integran los procesos gerenciales de planificación de objetivos y revisión del sistema ya que la definición de los objetivos del sistema de

Gestión Integrado abarca objetivos de calidad y de seguridad y salud en el trabajo y en las revisiones al sistema se toman en cuenta aspectos relacionados con las cuatro funciones, todo lo cual permite solucionar los conflictos de responsabilidades, y optimizar y simplificar el proceso de toma de decisiones basadas en datos integrales.

- Se alcanza una mejor coherencia, facilidad de manejo y reducción de los costos de mantenimiento de la documentación al integrarse en un solo documento (procedimiento, instrucción, registro, etc.) aspectos relacionados con las tres funciones.

- Se incrementa el rendimiento, las competencias y el entrenamiento de los miembros de la organización, ya que se determinan y suplen al unísono las necesidades de formación de los individuos en varias materias.

- Se logra un ahorro de recursos en el desarrollo e implementación del Sistema de Gestión Integrado y una menor inversión que la necesaria para los procesos de certificación de estos sistemas independientemente.

- Se pueden auditar al mismo tiempo los dos sistemas, con lo cual se reducirán los costos que para una organización supone la preparación y realización de dichas auditorías.

Sin embargo también tiene desventajas como pueden ser:

- Mayor costo de implantación respecto a un solo sistema particular de gestión. Si es difícil en cualquier organización poner a rodar uno de estos sistemas, hay que pensar lo que sería implantar los tres a la vez. Por esto la tónica actual de las organizaciones es implantar un Sistema de Gestión de la Calidad y posteriormente ir integrando los demás.

- Mayor esfuerzo en materia de formación, organización y de cambio de la cultura empresarial, al requerir la realización de un mayor número 
de actividades para garantizar la formación del personal en las varias materias.

- Se requiere de mayor esfuerzo en la planificación, el control de los procesos y en la toma de decisiones, ya que se deben tener en consideración aspectos relacionados con la calidad, el desempeño ambiental, el desempeño en seguridad y salud en el trabajo y la seguridad de la información en los procesos.

\section{RESULTADOS}

En el área de la calidad, también la Revolución Industrial cambia radicalmente los modelos empresariales que habían perdurado a través de los siglos. La masificación de las empresas, la división del trabajo, los operarios a destajo, hace que los sistemas tradicionales no sirvan para esta nueva situación.

A finales del XIX, el sistema de "Gestión científica" de Taylor y el sistema de "Producción en masa" de Ford, con la separación de la planificación de la producción, producen un aumento de la productividad y la disminución de la calidad de los productos. Esto provoca enormes problemas, que aconsejan el análisis estadístico de los defectos de los productos, con objeto de cuantificar la magnitud del problema.

Durante la Segunda Guerra Mundial, el ejército de los Estados Unidos de América, aplica los Military Standard, una serie de especificaciones técnicas a cumplir por los productos que compra. Aparece un nuevo concepto de Calidad como equiparable a "Conformidad a unas especificaciones".

Pero una cosa es lo que se pueda exigir a un producto en una recepción y otra es su comportamiento a lo largo de un periodo de tiempo en unas condiciones normales de uso. Se ve la necesidad de que el fabricante asegure que esto va a ser así y de esta forma en los años 50, se estudian técnicas de fiabilidad para asegurar la calidad de un producto durante su vida útil. Nace un nuevo concepto, el "aseguramiento de la calidad", mediante el cual se equipara a la calidad como "aptitud para el uso".

Pero todo cambia en los años 60, cuando en Japón se implanta una nueva forma de hacer las cosas, la calidad total, implantada con su modelo de gestión Total Quality Control (TQC), consiguiendo un éxito espectacular en el mundo. El modelo es copiado en los Estados Unidos de América, pero el resultado esperado no se consigue, por lo que necesita una adaptación que se plasma en el modelo Total Quality Management (TQM). La Unión Europea se mueve en esta dirección y crea en 1988 el modelo European Foundation for Quality Management (EFQM).

Una larga evolución que ha ido creando a lo largo de los años diferentes conceptos y formas de entender la Calidad. Desde el concepto inicial de Control del Proceso de fabricación (control durante el proceso de fabricación basado fundamentalmente en el control estadístico de procesos), se pasa al Control de Calidad (conjunto de actividades de inspección y ensayos encaminados a comprobar que el producto final cumple con las especificaciones), para llegar a la Garantía de Calidad 
(conjunto de actividades planificadas y sistemáticas, necesarias para garantizar que un producto o servicio va a satisfacer los requisitos de la calidad exigidos, independientemente del grado de inspección final que se aplique).

Finalmente se pasa a la Calidad Total (conjunto de principios y métodos, organizados dentro de una estrategia global, con vistas a movilizar a toda la empresa para lograr una mayor satisfacción del cliente interno y externo, al menor costo). En esta última fase, la Calidad de las prestaciones, del trabajo, de la organización, de la imagen, de cada puesto de trabajo, de las relaciones, la calidad como satisfacción del cliente y como resultado, hacen que las empresas que adoptan este modelo, logren los mejores resultados empresariales que se puedan esperar. Es el camino hacia la excelencia empresarial, camino que no se puede improvisar.

En nuestros días, la Gestión de la Calidad (ISO 9001:2008 - ISO 9001:2015) caracterizada por su impacto estratégico en las organizaciones como oportunidad de ventaja competitiva, afecta a la sociedad en general y es considerada una filosofía, una cultura, una estrategia y un estilo de gerencia de la empresa.

Mientras la Seguridad y Salud en el Trabajo ha pasado de un enfoque reactivo en los años 70 a un enfoque preventivo en nuestros días. Haciendo un poco de historia se puede asegurar que durante siglos, las actuaciones a favor de la protección de la salud de los trabajadores se mantienen en valores testimoniales. La revolución industrial del siglo XIX, trae un aumento considerable de los riesgos industriales, de los accidentes y de las enfermedades profesionales. Se producen actuaciones marcadas por un carácter proteccionista y reparador, dirigido a aquellos trabajadores que han sufrido un daño, y especialmente una lesión corporal motivada por un accidente de trabajo. Es la clásica protección de riesgos laborales, con actuaciones fundamentalmente reactivas o posteriores a la producción del daño.

La prevención activa comienza con H. Willians Heinrich, en 1931, al realizar un estudio sobre 70.000 accidentes de trabajo, obteniendo resultados convincentes acerca de la importancia que tiene para la empresa el estudio económico de los costos ocasionados por un accidente de trabajo, incluso si este no causa lesiones a un trabajador. Considera que la actuación más interesante es sobre los actos o condiciones peligrosas.

En 1969 Frank Bird estudia 1.753.498 casos de accidentes notificados en los Estados Unidos de América, concluyendo que el 5\% de la facturación de las empresas, se lo llevan los costes ocasionados por los accidentes, lo que le lleva a aconsejar que la gestión de la prevención debe integrarse en el sistema de gestión global de la empresa.

Actualmente las políticas preventivas, la negociación sindical, los costos derivados de los accidentes, incluidas las responsabilidades derivadas, la política de los seguros, la opinión de los consumidores y otros, son aspectos que deberían ser suficientes para la implantación por parte de las empresas de sistemas eficaces de gestión de riesgos.

Pasan los años, mejoran las condiciones de trabajo en algunas empresas, pero en la actualidad las estadísticas de la mayoría de los países 
desarrollados, nos golpean con datos desalentadores. Cada vez hay más accidentes de trabajo y enfermedades profesionales.

La conclusión es que falla el sistema, al no integrarse la gestión de la prevención de riesgos laborales en el sistema de gestión de la empresa.

La unión de los países en bloques para favorecer las relaciones entre ellos, permite fijar una serie de políticas comunes, que repercuten sobre el intercambio de trabajadores, productos y el medio ambiente, aportando herramientas que contribuyen a la implantación y mejora en los sistemas de gestión.

Así ha ocurrido en la Unión Europea (UE), con la gran reforma del Tratado Constitutivo de la Comunidad Europea mediante el Acta Única Europea de 1986, permitiendo que la Seguridad y Salud en el Trabajo se convierte en un tema prioritario, con las disposiciones relativas a Política Social de protección del trabajador [1] y a Política Interior de libre circulación de personas, productos, servicios y capitales. Aunque podría pensarse que ambas políticas tienen pocos vínculos en común, nada más alejado de la realidad, porque ambas políticas confluyen y se complementan en beneficio de una mayor prevención de riesgos para el trabajador. Por un lado la política social actúa sobre la mejora de las condiciones de trabajo del trabajador y la política interior tiene una influencia decisiva sobre la seguridad de los productos, equipos de trabajo e instalaciones industriales.

En un sistema de gestión integrada, el empresario deberá asumir que la prevención de los riesgos laborales deberá realizarse de forma permanente, mediante la integración de la actividad preventiva en el sistema general de gestión de la empresa, tanto en el conjunto de sus actividades como en todos los niveles jerárquicos de ésta. Adoptará cuantas medidas sean necesarias, en materia de plan de prevención de riesgos laborales, evaluación de riesgos, información, consulta, participación y formación de los trabajadores, actuación en casos de emergencia y de riesgo grave e inminente, vigilancia de la salud, y mediante la constitución de una organización y de los medios necesarios.

En 1999 se aprueba la norma OHSAS 18001:"Sistemas de Gestión de la Seguridad y Salud en el Trabajo. Especificación", (revisada en 2007) [2], con objeto de facilitar a las empresas a la implantación de un modelo de gestión preventiva, controlar los riesgos de seguridad y salud en el trabajo y mejorar su desempeño. Los requisitos de esta norma se han previsto para su incorporación a cualquier sistema de gestión, dependiendo la extensión de su aplicación de la política empresarial, la naturaleza de sus actividades, los riesgos y la complejidad de las operaciones. Para ayudar a su implementación en la empresa, se aprueba la norma OHSAS 18002:1999 "Sistemas de Gestión de la Seguridad y Salud en el Trabajo. Directrices para la implementación de OHSAS 18001" [3]. Es importante la correspondencia que establece entre OSHAS 18001, ISO 14001 de gestión ambiental [4] e ISO 9001 de gestión de la calidad [5]. Sin embargo, la reciente publicación de la ISO 45001:2018 facilita aún más esta integración al sistema de gestión de la calidad. 
Luego entonces, las primeras experiencias en integración de sistemas de gestión tienen lugar primero con la gestión del medio ambiente y posteriormente con la seguridad y salud en el trabajo.

Claves para una correcta integración de los sistemas de gestión Calidad - Seguridad y Salud en el Trabajo

Considerando que la ISO 9001:2015 [6] y la ISO 45001:2018 [7] tienen una estructura de alto nivel que posibilita la compatibilidad entre los dos sistemas de gestión en cuanto a la unidad en el vocabulario y los requisitos, esto facilita la integración. La estructura de ambas normas se basa en el Ciclo de Mejora PHVA y se sustenta en los principios siguientes:

- Enfoque al cliente

- Liderazgo

- Participación del personal

- Enfoque basado en procesos

- Mejora

- Toma de decisiones basadas en evidencias

- Gestión de las relaciones

Asimismo, se construyó en el Enfoque de Riesgo ya que el enfoque de su búsqueda es: mejorar la confianza y satisfacción del cliente y las partes interesadas; establecer una cultura proactiva, de prevención, mejora y protección y asegurar la consistencia de calidad de los productos y servicios.

Entonces, los pasos para una correcta integración de los sistemas de gestión de Calidad - Seguridad y Salud en el Trabajo a observar son:

1. Analizar si estos sistemas ya están implantados por separado o si la organización va a implantar un sistema integrado de gestión cual es la situación actual.

2. Nombrar una persona responsable de este proyecto de integración y crear un equipo de trabajo

3. Planificar las actividades a desarrollar, el cronograma de ejecución, los responsables y la documentación necesaria

4. Comunicar a todo el personal de la organización lo que se quiere hacer

Surge entonces una pregunta, ¿cuáles son los elementos comunes de ambas normas que facilitan la integración?

Estos elementos son:

- Consideración del contexto de la organización

- Liderazgo

- Planificación

- Soporte

- Operación

- Evaluación del desempeño

- Mejora

Considerando estos elementos un análisis de la estructura de estas normas permite conocer cómo se relacionan con el Ciclo de Mejora PHVA según se muestra a continuación en la tabla 1. 
Tabla 1.

Capítulos de las normas y su relación con los elementos comunes a ambos sistemas de gestión.

\begin{tabular}{|l|l|}
\hline Ciclo de Mejora & Capítulos \\
\hline \multirow{3}{*}{ PLANEAR } & 4. Contexto de la organización \\
\cline { 2 - 2 } & 5. Liderazgo \\
\cline { 2 - 2 } & 6. Planificación \\
\hline HACER & 7. Soporte \\
\cline { 2 - 2 } & 8. Operación \\
\hline VERIFICAR & 9. Evaluación del desempeño \\
\hline MEJORA & 10. Mejora \\
\hline
\end{tabular}

Los Autores

Se recomienda tener información documentada relativa a:

- Alcance del sistema integrado

- La necesaria para apoyar la operación

- Política integrada

- Objetivos

- Evidencia del desarrollo de los procesos

Mientras no se considera obligatorio tener información documentada relativa a:

- Manual

- Aplicar el 100\% de las normas

- Tener un representante del sistema integrado de gestión

- Diseñar un plan estratégico

- Realizar acciones correctivas para cada no conformidad

Sin dudas, es relevante las ventajas que supone la integración de los sistemas de gestión de Calidad - Seguridad y Salud en el Trabajo pero dos aspectos importantes a considerar para facilitar la implementación de un sistema integrado de gestión son las competencias del personal y su formación. Por tanto demanda cambios en la Gestión del Talento Humano en las organizaciones. Esto implicaría la Gestión por Competencias en las organizaciones que decidan la adopción de sistemas integrados de gestión, en este caso específico Calidad - Seguridad y Salud en el Trabajo.

\section{DISCUSIÓN}

La estructura de alto nivel de la ISO 9001:2015 y la ISO 45001:2018 para la Gestión de la Calidad y la Seguridad y Salud en el Trabajo respectivamente posibilita la compatibilidad entre los dos sistemas ya que existen elementos comunes por lo que su integración es una alternativa posible y recomendada para aumentar la eficacia de las organizaciones.

El Sistema Integrado de Gestión de la Calidad y la Seguridad y Salud en el Trabajo basado en el enfoque de riesgos permite mejorar la confianza y satisfacción del cliente y las partes interesadas; establecer una cultura proactiva, de prevención, mejora y protección y asegurar la consistencia de calidad de los productos y servicios. 
El éxito en la implementación del Sistema Integrado de Gestión de la Calidad y la Seguridad y Salud en el Trabajo dependerá en gran medida la Gestión por Competencias del Talento Humano en las organizaciones. El conocimiento, la formación continua, la educación y el compromiso de los trabajadores serán factores claves para que las empresas puedan competir, crecer y seguir evolucionando, en un mercado cada vez más competitivo.

\section{Referencias}

[1] Unión Europea. Directiva Marco 89/391 CEE del Consejo, de 12 de junio de 1989, relativa a la aplicación de medidas para promover la mejora de la Seguridad y de la Salud de los trabajadores en el trabajo DOCE L 183 de 29-6- 1989.

[2] OSHAS 18001:2007. Sistemas de Gestión de la Seguridad y Salud en el Trabajo.

[3] OSHAS 18002:2008. Sistemas de Gestión de la Seguridad y Salud en el Trabajo. Directrices para la implementación de OHSAS 18001:2007.

[4] ISO 14001:2004. Sistemas de Gestión Medioambiental. Especificaciones y directrices para su utilización.

[5] ISO 9001:2008. Sistemas de Gestión de la Calidad. Requisitos.

[6] ISO 9001: 2015. Sistemas de Gestión de la Calidad. Requisitos.

[7] ISO 45001:2018. Sistemas de Gestión de la Seguridad y la Salud en el Trabajo. Requisitos con orientación para su uso. 